\title{
Disk emission and atmospheric absorption lines in black hole candidate 4U 1630-472
}

\author{
A. Różańska ${ }^{1}$, J. Madej $^{2}$, P. Bagińska ${ }^{1}$, K. Hryniewicz ${ }^{1}$, and B. Handzlik ${ }^{2}$ \\ 1 N. Copernicus Astronomical Center, Bartycka 18, 00-716 Warsaw, Poland \\ e-mail: agata@camk.edu.pl \\ 2 Warsaw University Observatory, Al. Ujazdowskie 4, 00-478 Warsaw, Poland \\ e-mail: jm@astrouw.edu.pl
}

Received 25 March 2013 / Accepted 2 December 2013

\begin{abstract}
Context. We analyzed Suzaku data of the black hole candidate 4U 1630-472 when it was in the high/soft state. The source, known for X-ray outbursts and for absorption dips, has an X-ray continuum spectrum that is interpreted correctly as emission from an accretion disk. Additionally, two absorption lines from $\mathrm{He}$ and $\mathrm{H}$-like iron have been clearly detected in the high-resolution data from 4U 1630-472.

Aims. We show that the continuum X-ray spectrum of $4 \mathrm{U}$ 1630-472 with iron absorption lines can be satisfactorily modeled by the spectrum from an accretion disk atmosphere. Absorption lines of highly ionized iron originating in a hot accretion-disk atmosphere can be an alternative or complementary explanation to the wind model usually favored for this type of sources.

Methods. We performed full radiative transfer calculations to model the emission from an accretion disk surface that is seen at different viewing angles using our transfer code ATM21. Computed models are then fitted to high-resolution X-ray spectra of 4U 1630-472 obtained by the Suzaku satellite.

Results. We modeled continuum and line spectra using a single model. Absorption lines of highly ionized iron can originate in the upper parts of the disk atmosphere, which is intrinsically hot because of the high disk temperature. Iron line profiles computed with natural, thermal, and pressure broadenings match observations very well.

Conclusions. According to any global disk models considered for the mass of central object that is close to $10 M_{\odot}$ or less, the effective temperature of the inner radii reaches $10^{7} \mathrm{~K}$. We showed that the accretion disk atmosphere can effectively produce iron absorption lines observed in 4U 1630-472 spectrum. Absorption line arising in an accretion disk atmosphere is the important part of the observed line profile, even if there are also other mechanisms responsible for the absorption features. Nevertheless, the wind theory can be an artefact of the fitting procedure when the continuum and lines are fitted as separate model components.
\end{abstract}

Key words. X-rays: binaries - stars: individual: 4U 1630-472 - accretion, accretion disks - line: profiles - line: formation

\section{Introduction}

Emission from the accretion disk around a compact object is the commonly accepted model for the soft X-ray bump observed in Galactic Black Hole candidates (GBHc). Depending on the mass of the central object and on the accretion rate, the inner disk temperature for the black hole of $10 M_{\odot}$ can be on the order of $10^{7} \mathrm{~K}$ (Różańska et al. 2011b). Any existing accretion disk theory - from a geometrically thin, optically thick standard disk (hereafter SS disk, Shakura \& Sunyaev 1973), even with relativistic corrections (hereafter NT disk, Novikov \& Thorne 1973), to a slim disk (Abramowicz et al. 1988) - gives the same temperature behaviour. The temperature at a given radius always increases with the decreasing mass of the central object and with the increasing accretion rate.

For such high temperatures for an accretion disk atmosphere, thermal lines from $\mathrm{H}$-like and He-like iron ions should be formed, and those lines should be visible between 6.7 to $9.2 \mathrm{keV}$, where the latter value is the energy of the last iron bound-free transition (from H-like ion to complete ionization). Nevertheless, it is well known that observed X-ray spectra from compact binaries do not always exhibit a disk component. Owing to instrument's technical limits and to spectral state transition, multi-temperature disk component may not be detected in full X-ray energy range. To explain data by the accretion disk emission, we have to be sure that observations were taken when the source was in the so-called "soft state", which is dominated by disk-like component.

Recently, several X-ray binaries have exhibited absorption lines from highly ionized iron (Boirin et al. 2004; Kubota et al. 2007; Díaz Trigo et al. 2012). Many of those sources show dips in their light curves, which are believed to be caused by obscuration of the central X-ray source by a dense material located at the outer edge of an accretion disk. Such obscuring material was accumulated during the accretion phase from the companion star onto the disk (White \& Swank 1982). The presence of dips and the lack of total X-ray eclipses by the companion star indicate that the system is viewed relatively close to edge-on, at an inclination angle in the range $\sim 60-80^{\circ}$ (Frank et al. 1987).

The He-like and $\mathrm{H}$-like $\mathrm{Fe}$ absorption features indicate that highly ionized plasma is present in these systems. Study of these lines is extremely important for characterizing the geometry and physical properties of plasma. Recently, it has been shown that the presence of absorption lines is not necessarily related to the viewing angle since non-dipping sources also show those features (Díaz Trigo et al. 2012). Moreover, the Fe XXV absorption line was also observed during non-dipping intervals in XB1916-053 (Boirin et al. 2004). 
The usual way to model highly ionized Fe absorption lines from those sources is at first to fit continuum to the spectrum, given either by multiblackbody disk emission or by a powerlaw shape. After fitting of the continuum each line is fitted separately using a Gaussian shape. Equivalent widths (EWs) and velocity shifts of the line centroid can be derived this way. In most cases, absorbing material is outflowing with velocities reaching a few hundred $\mathrm{km} \mathrm{s}^{-1}$. The extreme example is the recent observation of black hole candidate IGR J17091-3624, where the wind velocity was estimated as 0.03 c (King et al. 2012). Additionally, photoionization modeling can be done using a grid of models computed by XSTAR (Kallman \& Bautista 2001) if the quality of spectrum is good enough. Nevertheless, this is always done after continuum fitting is established, and usually at least two or more photoionized slab components are needed to model all lines (King et al. 2012). For the black hole transient IGR J17091-3624, EWs of Fe XXV and Fe XXVI lines are approximately twice bigger when fitting XSTAR model, than for only the Gaussian shape.

In this paper, we present analysis of Suzaku XIS X-ray spectra of $4 \mathrm{U}$ 1630-472 alternative to the one presented in Kubota et al. (2007). Instead of multiblackbody disk emission DISKBB with two Gaussians fittet separately, we used our atmospheric disk emission models (ATM) recently computed and published in Różańska et al. (2011b). Our theoretical spectra were obtained from careful radiative transfer computations in accretiondisk atmospheres. All calculations of continuum and line spectra were performed simultaneously, taking relativistic Compton scattering of X-ray photons into account. Absorption profiles of Fe XXV and Fe XXVI spectral lines were carefully computed as a convolution of natural, thermal (Gaussian), and pressure broadenings. Expressions describing pressure broadening of iron lines were deduced from considerations by Griem (1974). In the case of our models, lines are fitted together with underlying continuum.

The paper is organized as follows. Section 2 describes the recent observational status of Fe absorption lines in X-ray binaries, and Sect. 3 explains the principal assumptions of our theoretical model. All the procedures of data reduction and spectral fitting, which were done by us are presented in Sect. 4. Conclusions and discussion are formulated in Sect. 5.

\section{Narrow Fe absorption lines in X-ray binaries}

Different types of X-ray binaries exhibit the presence of narrow absorption lines from highly ionized iron. Table 1 is an updated version of the Table 5 from Boirin et al. (2004). In the past research, most objects were fitted using POWERLAW model to describe a continuum and a Gaussian shape for lines. In some sources, being in soft state (i.e., dominated by the disk component) multi-temperature disk black body model (DISKBB), and Voigt profiles for individual lines (KABS) were considered (Kubota et al. 2007).

Gaussian profile fitted to an individual absorption line puts constrain on the ionic column density. These ionic column densities can be incorporated into photoionization calculations to derive the ionization parameter and total column density of absorbing material. In the case of X-ray binaries, photoionization models show $N_{\mathrm{H}}$ on the order of $10^{23-24} \mathrm{~cm}^{-2}$ with the value of ionization parameter $\xi=10^{3-4}$. Such estimates were obtained for a few systems that are assumed to be highly inclined.

Usually prominent $\mathrm{K}_{\alpha}$ absorption lines from $\mathrm{He}$ and $\mathrm{H}$-like iron are detected with EWs from -5 up to $-61 \mathrm{eVs}$ (minus sign denotes an absorption line). Only in few systems are $\mathrm{K}_{\beta}$ lines visible. The extreme case is XB1916-053, where He-like iron line changes its EW during the persistent and dipping time intervals. In the persistent phase, $E W=-30_{-12}^{+8}$, while during the dipping phase the EW changes from $E W=-74_{-27}^{+20}$ to $E W=-168_{-46}^{+44}$ (Boirin et al. 2004). A summary of the objects with absorption lines and the connection of EWs with spectral state is shown in Ponti et al. (2012).

In GX $13+1$, properties of the absorber do not vary strongly with the orbital phase, which suggests that ionized plasma has a cylindrical geometry. Therefore, inclination of GX $13+1$ was constrained between $60-80^{\circ}$. Almost all values of inclinations in the fourth column of Table 1 were not known before the discovery of iron absorption lines in those sources. After adopting wind geometry, the presence of $\mathrm{Fe}$ absorption lines suggests high inclination for those sources. This is why inclination numbers in the fourth column are so similar.

Properties of the absorption features in GX $13+1$ and MXB 1659-298 show no obvious dependence on the orbital phase, except during a dip from X1624-490, where there is evidence of additional colder material. Boirin \& Parmar (2003) have modeled the narrow absorption features of Fe XXVI $\mathrm{K}_{\alpha}$ and $\mathrm{K}_{\beta}$ during non-dip intervals, therefore, ionized absorption features may be common characteristics of disk accreting systems. Systems with shorter orbital periods are expected to have smaller accretion disks.

In XB 1323-619 a number of absorption lines (6.7 and $6.9 \mathrm{keV}$ ) were discovered in non-dip, non-burst spectra (Church et al. 2005). Curve-of-growth analysis has provided a consistent solution in which a line ratio was reproduced by assuming collisional ionization with $k T=31 \mathrm{keV}$, close to the accretion disk corona (ADC) electron temperature in this source. Thus, Church et al. (2005) proposed that those absorption lines in the dipping low-mass X-ray binaries (LMXB) are produced in the ADC.

From an observational point of view, ADC can produce the same spectral features as the upper layers of the accretion disk atmosphere. The temperatures of both emission regions can be comparable as we explain in the following sections.

\section{Fe absorption lines in accretion disk atmosphere}

In this paper, we fit the few iron-rich model disk atmospheres, which were already computed and presented in Różańska et al. (2011b) to the Suzaku X-ray spectra of 4U 1630-472. Since the observed iron lines in 4U 1630-472 were discovered in absorption, we only used non-irradiated modeled disk spectra.

Full simulation of emission from accretion disk atmospheres is very time consuming and depends on the assumed global disk model. The code used in this paper was described fully in our previous paper (Różańska et al. 2011b). We call here the most essential properties of our numerical approach. The accretion disks at a given accretion rate and black hole spin are divided onto approximately 15 rings. At each ring, we computed a local model atmosphere using complete and consistent radiative transfer code ATM21 (Różańska \& Madej 2008). The code assumes that the vertical optical depth at some standard photon frequency is the independent variable in the model. At the fixed chemical composition, the model atmosphere assumed two parameters: the effective temperature, which at each ring was determined from the accretion rate, and the gravity in a vertical direction caused by the black hole for the given mass. Each model atmosphere was definitely not gray, and the local emissivity or opacity 
Table 1. Measured absorption line properties and orbital parameters of sources with observed Fe absorption lines.

\begin{tabular}{|c|c|c|c|c|c|c|c|c|}
\hline Source & Ref. & Type & $P_{\text {orb }}(h)$ & $i\left(^{\circ}\right)$ & $\begin{array}{l}\text { Fe XxV K } \alpha \\
E(\mathrm{keV}) \\
E W(\mathrm{eV})\end{array}$ & $\begin{array}{l}\text { Fe XXVI K } \alpha \\
E(\mathrm{keV}) \\
E W(\mathrm{eV})\end{array}$ & $\begin{array}{l}\text { Fe XXV K } \beta \\
E(\mathrm{keV}) \\
E W(\mathrm{eV})\end{array}$ & $\begin{array}{l}\text { Fe XXVI K } \beta \\
E(\mathrm{keV}) \\
E W(\mathrm{eV})\end{array}$ \\
\hline \multirow[t]{2}{*}{ XB 1916-053 (NS) } & (1) & LMXB & 0.8 & $60-80$ & $6.65_{-0.02}^{+0.05}$ & $6.95_{-0.04}^{+0.05}$ & $7.82_{-0.07}^{+0.03 a}$ & $8.29_{-0.12}^{+0.08}$ \\
\hline & & $\mathrm{D}, \mathrm{B}$ & & & $-30_{-12}^{+8}$ & $-30_{-12}^{+11}$ & $-21_{-12}^{+9}$ & $-19_{-12}^{+11} \mathrm{eV}$ \\
\hline \multirow[t]{2}{*}{ GX 13+1 (NS) } & (2) & LMXB & 577.4 & $60-80^{b}$ & $6.75_{-0.05}^{+0.02}$ & $7.02 \pm 0.06$ & $7.91_{-0.15}^{+0.46}$ & $8.24_{-0.06}^{+0.04}$ \\
\hline & & Atol, B & & & $-20 \pm 5$ & $-41 \pm 7$ & $-33 \pm 12$ & $-37 \pm 15$ \\
\hline \multirow[t]{2}{*}{ MXB 1659-298 (NS) } & (3) & LMBX & 7.1 & $\sim 80$ & $6.64 \pm 0.02$ & $6.90_{-0.01}^{+0.02}$ & - & - \\
\hline & & $\mathrm{D}, \mathrm{E}, \mathrm{T}$ & & & $-33_{-20}^{+9}$ & $-42_{-13}^{+8}$ & - & - \\
\hline \multirow[t]{2}{*}{ X 1254-690 (NS) } & (4) & LMXB & 3.9 & $60-80$ & - & $6.95 \pm 0.03$ & - & $8.20_{-0.10}^{+0.05}$ \\
\hline & & $\mathrm{D}$ & & & - & $-27_{-8}^{+11}$ & - & $-17 \pm 9$ \\
\hline \multirow[t]{2}{*}{ X 1624-490 } & (5) & LMXB & 20.9 & $60-80$ & $6.72 \pm 0.03$ & $7.0 \pm 0.02$ & - & - \\
\hline & & $\mathrm{D}$ & & & $-7.5_{-6.3}^{+1.7}$ & $-16.6_{-5.9}^{+1.9}$ & - & - \\
\hline \multirow[t]{2}{*}{ XB 1323-619 } & (6) & LMXB & 2.93 & $60-80$ & $6.70 \pm 0.03$ & $6.98 \pm 0.04$ & - & - \\
\hline & & $\mathrm{D}, \mathrm{qB}$ & & & $-22 \pm 3$ & $-27 \pm 4$ & - & - \\
\hline Cir X-1 (NS) & (7) & $\mathrm{B}, \mathrm{D}, \mathrm{O}, \mathrm{J}$ & 398 & high & $6.701 \pm 0.009$ & $6.965 \pm 0.01$ & - & - \\
\hline \multirow[t]{2}{*}{ 4U 1630-472 (BH) } & (8) & LMXB & 690 & $\sim 70$ & $6.72 \pm 0.01$ & $6.980_{-0.007}^{+0.009}$ & $7.84_{-0.03}^{+0.04}$ & $8.11_{-0.06}^{+0.05}$ \\
\hline & & $\mathrm{D}, \mathrm{T}, \mathrm{O}$ & & & $-21 \pm 3$ & $-30_{-3}^{+2}$ & $-18.2_{-6}^{+4}$ & $-15.8_{-7}^{+5}$ \\
\hline \multirow[t]{4}{*}{ GRO J1655-40 (BH) } & (9) & microq & $62.9^{c}$ & $69-85$ & $6.63 \pm 0.07$ & $6.95 \pm 0.10$ & $7.66 \pm 0.13$ & - \\
\hline & & $\mathrm{D}, \mathrm{O}$ & & & $-61_{-13}^{+15}$ & $-25_{-11}^{+13}$ & $-35_{-29}^{+30}$ & - \\
\hline & $(10)$ & & & & $6.698 \pm 0.001$ & $6.999 \pm 0.002$ & $7.842 \pm 0.005$ & $8.277 \pm 0.01$ \\
\hline & & & & & $-45.59 \pm 1.8$ & $-30.82 \pm 2.37$ & $-48.11 \pm 4.46$ & $-27.63 \pm 5.53$ \\
\hline \multirow[t]{4}{*}{ GRS 1915+105 (BH) } & (11) & microq & 804 & $\sim 70$ & $6.72_{-0.017}^{+0.015}$ & $7.000_{-0.014}^{+0.020}$ & $7.843_{-0.035}^{+0.037}$ & $8.199_{-0.064}^{+0.065}$ \\
\hline & & & & & $-35.8_{-7.8}^{+3.7}$ & $-43.2_{-5.3}^{+6.3}$ & $-30.8_{-5.8}^{+11.2}$ & $-22 \cdot_{-12}^{+11}$ \\
\hline & (12) & & & & $6.684 \pm 0.022$ & $6.984 \pm 0.009$ & - & - \\
\hline & & & & & & -18 & - & - \\
\hline \multirow[t]{2}{*}{ H1743-322 (BH) } & (13) & microq & - & $\sim 70$ & $6.709 \pm 0.004$ & $6.981 \pm 0.004$ & - & - \\
\hline & & $\mathrm{D}, \mathrm{T}, \mathrm{O}$ & & & $-5 \pm 1$ & $-20 \pm 2$ & - & - \\
\hline \multirow[t]{2}{*}{ IGR J17091-3624 (BH) } & (14) & $\mathrm{T}, \mathrm{O}$ & - & - & $6.91 \pm 0.01$ & $7.32_{-0.06}^{+0.01}$ & - & - \\
\hline & & & & & $-21_{-0.2}^{+0.5}$ & $-32_{-0.4}^{+1.8}$ & - & - \\
\hline
\end{tabular}

Notes. L - LMXB; microq - micro-quasars; D - deeps; E - eclipses; B - bursts; qB - quasi-periodic bursts; T - transient; O - outburst; J - radio jet. ${ }^{(a)}$ Identified as S XVI and Ni XXVII transitions by Boirin et al. (2004). ${ }^{(b)}$ Low $i$ suggested by high radio to X-ray ratio (Schnerr et al. 2003), but high $i \simeq 60-80^{\circ}$ by detection of Fe abs. lines (Díaz Trigo et al. 2012). ${ }^{(c)}$ From optical observations (Orosz \& Bailyn 1997).

References. (1) XMM, Boirin et al. (2004); (2) XMM, Ueda et al. (2001); Díaz Trigo et al. (2012); (3) XMM, Sidoli et al. (2001); (4) XMM, Boirin \& Parmar (2003); (5) XMM, Parmar et al. (2002); (6) XMM, Church et al. (2005); (7) Chandra; Brandt \& Schulz (2000); (8) Suzaku epoch 4, Kubota et al. (2007); (9) ASCA, Ueda et al. (1998); (10) Chandra, Miller et al. (2008); (11) ASCA, Kotani et al. (2000); (12) Chandra, Lee et al. (2002); (13) Chandra epoch 1, Miller et al. (2006); (14) Chandra, King et al. (2012).

was a sum of non-gray bound-free, free-free, and bound-bound absorption or emission caused by individual ions. Moreover, local opacity and emissivity were influenced by Compton scattering by free electrons. We always assumed that a single local electron temperature is valid for all processes, also those that include ions. Velocity distribution of all particles is relativistic and Maxwellian.

We numerically simulated emission by assuming a radial effective temperature distribution for the slim disk model (Abramowicz et al. 1988) as the most reasonable one. Nevertheless, calculations of $T_{\text {eff }}(R)$ for the standard SS disk
(Shakura \& Sunyaev 1973) or for the NT disk (Novikov \& Thorne 1973) give similar results, as shown in Fig. 1 of Różańska et al. (2011b). In all cases, for the mass of black hole $M_{\mathrm{BH}}=10 M_{\odot}$ and for a high accretion rate, the temperature in the inner disk can reach $10^{7} \mathrm{~K}$. This temperature even rises when the spin of the black hole increases.

The whole disk was divided into individual rings, and at each distance from the black hole full radiative transfer computations were done assuming that the atmosphere is in radiative and hydrostatic equilibrium. We used our code for model atmosphere computations, ATM21, suitable for computing disk vertical 
Table 2. Equivalent widths of the model iron line components (in eV): Fe XXV and Fe XXVI resonant lines for five sets of the couple of parameters $\dot{m}$ and spin $a$.

\begin{tabular}{|c|c|c|c|c|c|c|c|c|c|c|}
\hline inc. $\left({ }^{\circ}\right)$ & $\begin{array}{c}\dot{m}=0.01 \\
\mathrm{Fe} X X V\end{array}$ & $\begin{array}{l}a=0.98 \\
\text { Fe XXVI }\end{array}$ & $\begin{array}{l}\dot{m}=0.1 \\
\mathrm{Fe} X \mathrm{XV}\end{array}$ & $\begin{array}{l}a=0.98 \\
\text { Fe XXVI }\end{array}$ & $\begin{array}{c}\dot{m}=0.01 \\
\mathrm{FeXXV}\end{array}$ & $\begin{array}{l}a=0 \\
\text { Fe XXVI }\end{array}$ & $\begin{array}{l}\dot{m}=0.1 \\
\text { Fe XXV }\end{array}$ & $\begin{array}{c}a=0 \\
\text { Fe XXVI }\end{array}$ & $\begin{array}{c}\dot{m}=1 \\
\text { Fe XXV }\end{array}$ & $\begin{array}{c}a=0 \\
\text { Fe XXVI }\end{array}$ \\
\hline 11.4 & -20.5 & -10.5 & -7.5 & -3.6 & -56.7 & -1.7 & -50.0 & -1.7 & -41.1 & -9.7 \\
\hline 26.1 & -20.1 & -10.3 & -7.4 & -3.5 & -54.7 & -1.6 & -49.4 & -1.8 & -40.8 & -9.5 \\
\hline 40.3 & -19.4 & -9.9 & -7.0 & -3.1 & -50.4 & -1.4 & -48.4 & -1.8 & -40.0 & -9.1 \\
\hline 53.7 & -18.2 & -9.3 & -6.6 & -2.7 & -44.0 & -1.1 & -46.8 & -1.9 & -38.5 & -8.8 \\
\hline 65.9 & -16.4 & -8.4 & -5.9 & -2.1 & -37.9 & -0.9 & -44.6 & -2.0 & -35.9 & -8.6 \\
\hline 76.3 & -13.8 & -7.5 & -4.9 & -1.6 & -34.7 & -0.8 & -41.6 & -2.2 & -31.7 & -8.9 \\
\hline 84.2 & -10.4 & -6.3 & -3.7 & -1.2 & -34.5 & -0.7 & -36.9 & -2.6 & -25.2 & -9.8 \\
\hline 88.9 & -6.8 & -4.7 & -2.4 & -1.1 & -36.5 & -0.6 & -26.9 & -4.3 & -15.4 & -10.6 \\
\hline
\end{tabular}

Notes. All EWs are given for viewing angles from $i=11.4^{\circ}$ (face-on disk), to $i=88.9^{\circ}$ (edge-on disk). In the headings we put global parameters of a model: accretion rate $\dot{m}$ in units of Eddington accretion rate, and dimensionless black hole spin $a$.

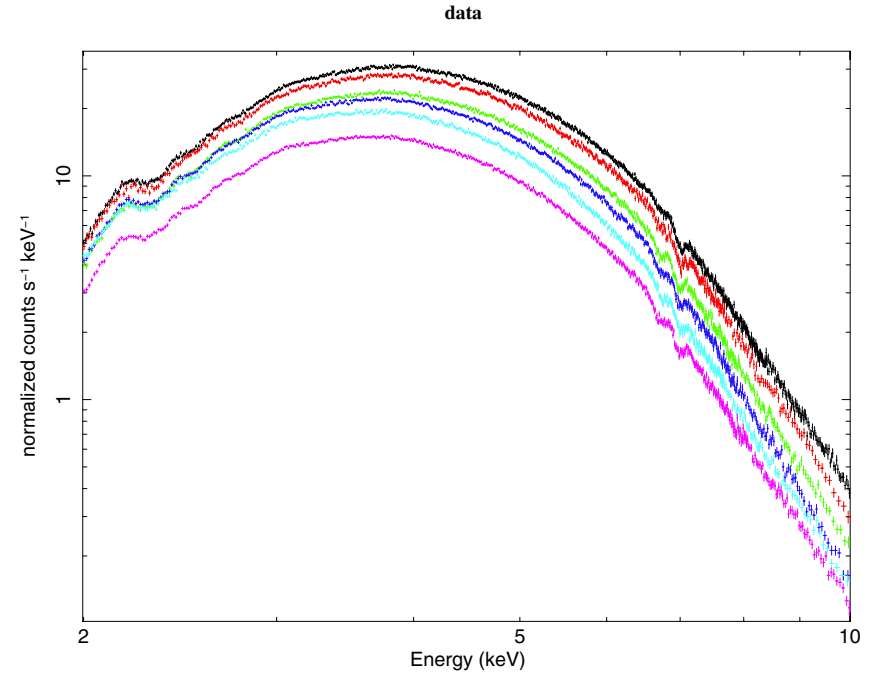

data

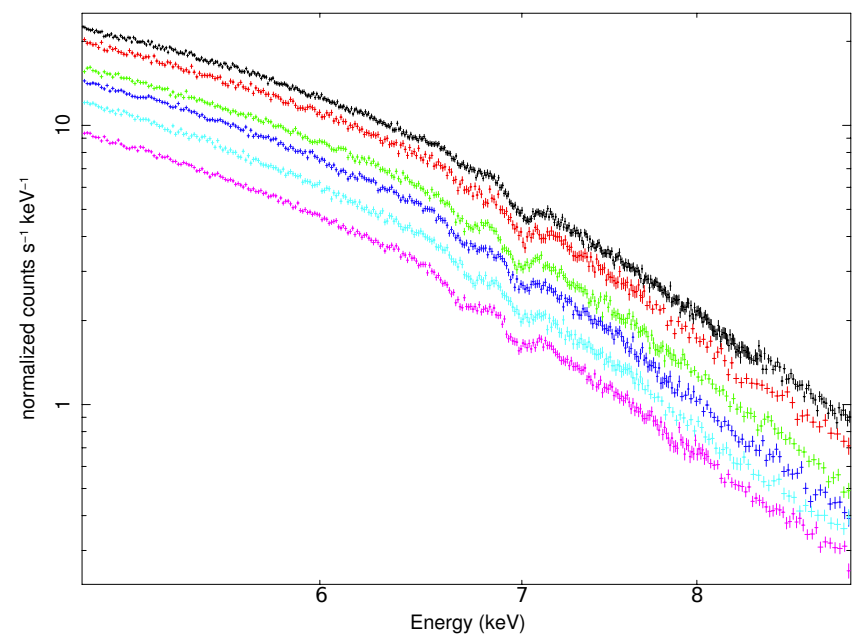

Fig. 1. Upper panel: X-ray spectra of $4 \mathrm{U} 1630-472$ obtained with the XIS023 showing a prominent bump similar to multi-temperature disk emission. Lower panel: focus on the iron line region from $5-9 \mathrm{keV}$, where absorption features are clearly seen. Colors mean different epochs of observations starting from black - epoch 1, up to magenta - epoch 6. The data are very similar to those presented in Kubota et al. (2007) in Figs. 2 and 5.

structure and its X-ray intensity spectra. Compton scattering on free electrons and ionization structure were self consistently taken into account (Madej \& Różańska 2004). The final spectrum of the disk was obtained by integrating contributions from individual rings.

In particular, our X-ray model spectra included profiles of the eight most prominent lines from highly ionized iron. Those lines belong to two fundamental series of iron. Helium-like iron Fe XXV produces the resonant line at $E=6690 \mathrm{eV}$, commonly denoted as $6.7 \mathrm{keV}$, and three lines at energies of $E=$ 7881,8226 , and $8416 \mathrm{eV}$. The series of hydrogen-like iron Fe XXVI consists of the resonant line at $E=6898 \mathrm{eV}$ and higher lines at $E=8172,8621$, and $8829 \mathrm{eV}$.

Additionally, we included three bound-free edges: Fe XXIV edge at $E=8690 \mathrm{eV}, \mathrm{FeXXV}$ edge at $E=8829 \mathrm{eV}$, and Fe XXVI edge at $E=9278 \mathrm{eV}$, which are visible in the highenergy X-ray band. These are ionization energies from the innermost K-shell. Other iron edges, which were included in our computations, arise from all K, L, M shells, but we do not list them since they are not prominent above $2 \mathrm{keV}$.

Różańska et al. (2011b) have shown that in galactic black hole binaries, accretion disk must be very hot, with local effective temperatures approaching $10^{7} \mathrm{~K}$ on the innermost orbits. In such high temperatures, iron is almost fully ionized, and spectral features of helium and hydrogen-like resonant iron lines must play a crucial role in interpreting the observed GBHB spectra in the soft state. Therefore, iron absorption lines, which were observed recently in disk-dominated binaries (see section above), most likely are resonant lines from the upper layers of accretion disk atmospheres. In our models we take all three line broadening mechanisms into account: natural, thermal (Gaussian) and pressure broadening. Broadened iron line profiles were carefully computed as the convolution of all three partial broadenings.

Table 2 presents theoretical equivalent widths of resonant lines computed in our radiative transfer simulations (Różańska et al. 2011b). All were computed only for five sets of global disk parameters and for eight viewing angles. In all cases, the mass of the black hole was assumed to be equal to $10 M_{\odot}$; nevertheless, we do not have any mass estimation in 4U 1630-472. Models do not cover all values in the parameter space, but in this paper we present fitting to the $4 \mathrm{U} 1630-472$ absorption lines for the first time together with underlying continuum.

Theoretical EWs are usually not very high, and the ratio of EWs for Fe XXV and Fe XXVI lines is on the order of 2 only for the hottest model (four leftmost columns in Table 2). For other models, the atmospheric temperature is too low to produce a prominent hydrogen-like iron line. In future work we plan to 
compute the whole grid of disk atmosphere models and make them suitable for spectral fitting.

\section{Suzaku observations of $4 \mathrm{U} 1630-472$}

\subsection{The source}

Black hole candidate 4U 1630-472 is known for X-ray outbursts that repeat within roughly 600-690 days (Jones et al. 1976; Parmar et al. 1995). The source has been observed by many major X-ray missions, showing heavy absorption with $N_{\mathrm{H}}=5-12 \times 10^{22} \mathrm{~cm}^{-2}$ (Tomsick et al. 1998). X-ray absorption dips observed in this source suggest a large inclination angle around $60^{\circ}$ (Kuulkers et al. 1998). This conclusion was based on the model where dips are produced during the eclipse of accretion disk corona (ADC) by a warm bulge, which develops when matter falls from a companion star onto the outer rim of the disk.

No optical counterpart is known for 4U 1630-472, mostly because of its high reddening and location in a crowded star field (Parmar et al. 1986). Therefore, the distance and mass of the compact object in 4U 1630-472 are unknown, and the source is classified as a black hole, owing to the similarity of its X-ray spectral and timing properties to those shown by systems with measured black hole masses.

Recent Suzaku monitoring of the source has led to successful detection of absorption lines in all six observations (Kubota et al. 2007). Lines were interpreted as an absorption in the wind. In this paper we show that this is not the only interpretation. By fitting iron absorption lines produced in outer regions of the accretion disk and hot atmosphere, together with underlying continuum, we demonstrate that existing satellites are not able to distinguish between both models. The model of outflowing wind with velocity on the order of $100 \mathrm{~km} \mathrm{~s}^{-1}$ fits, as does the model of static atmospheres. Our analysis also suggests that the wind can originate in a hot accretion disk atmosphere.

\subsection{Data reduction}

We downloaded and reduced the same Suzaku data of 4U 1630-472 as those published by Kubota et al. (2007). Six observations were performed in the time period from 08.02.2006 to 23.03.2006 after an outburst reported in 12.2005 by RXTE ASM. The exact time of observations made by Suzaku in comparison with an ASM lightcurve is marked in Fig. 1 (Kubota et al. 2007) by vertical arrows.

The source was observed by X-ray Imaging Spectrometer (XIS) operating in the $0.2-12 \mathrm{keV}$ energy range, and one of two hard X-ray detectors (HXD), both instruments onboard Suzaku. Nevertheless, for this analysis we took only XIS data. XIS is built out of four CCDs, located in the focal plane of X-ray mirrors (XRT). Three of those CCDs, named XIS0, XIS2, and XIS3, are front-illuminated (FI), while the XIS1 sensor is backilluminated (BI). In this paper we analyze both FI and BI CCD data. Owing to high brightness of 4 U 1630-472, XIS was set to one-quarter window option (which means we have $2 \mathrm{~s}$ read-out time), and the first four observations were performed in burst option. The $2 \times 2$ and $3 \times 3$ editing modes were used in observations. All exposure times and count rates are presented in Table 3.

In further analysis we used only $3 \times 3$ editing mode data because $2 \times 2$ data were not well calibrated during the observation time. Although background was negligible during all six observations, we subtracted it from the data. For extracting spectra
Table 3. Suzaku observations of $4 \mathrm{U} 1630-472$ for all six epochs of year 2006.

\begin{tabular}{|c|c|c|c|}
\hline Epoch & XIS & $T_{\exp }[\mathrm{ks}]$ & Count rate \\
\hline & 0 & 11.08 & $8.87 \mathrm{E}+01 \pm 8.95 \mathrm{E}-02$ \\
\hline 1 & 1 & 11.06 & $8.79 \mathrm{E}+01 \pm 8.92 \mathrm{E}-02$ \\
\hline \multirow[t]{5}{*}{ Feb. 8} & 2 & 11.02 & $1.09 \mathrm{E}+02 \pm 9.94 \mathrm{E}-02$ \\
\hline & 3 & 11.08 & $1.28 \mathrm{E}+02 \pm 1.08 \mathrm{E}-01$ \\
\hline & FI & 33.17 & $1.02 \mathrm{E}+02 \pm 1.10 \mathrm{E}-01$ \\
\hline & $\mathrm{FI}+\mathrm{BI}$ & 44.23 & $9.86 \mathrm{E}+01 \pm 7.60 \mathrm{E}-02$ \\
\hline & 0 & 4.79 & $7.48 \mathrm{E}+01 \pm 1.25 \mathrm{E}-01$ \\
\hline 2 & 1 & 4.86 & $7.37 \mathrm{E}+01 \pm 1.23 \mathrm{E}-01$ \\
\hline \multirow[t]{5}{*}{ Feb. 15} & 2 & 4.76 & $9.06 \mathrm{E}+01 \pm 1.38 \mathrm{E}-01$ \\
\hline & 3 & 4.79 & $8.95 \mathrm{E}+01 \pm 1.37 \mathrm{E}-01$ \\
\hline & FI & 14.34 & $8.41 \mathrm{E}+01 \pm 8.09 \mathrm{E}-02$ \\
\hline & $\mathrm{FI}+\mathrm{BI}$ & 19.20 & $8.13 \mathrm{E}+01 \pm 6.84 \mathrm{E}-02$ \\
\hline & 0 & 10.7 & $5.73 \mathrm{E}+01 \pm 7.32 \mathrm{E}-02$ \\
\hline 3 & 1 & 10.71 & $5.84 \mathrm{E}+01 \pm 7.38 \mathrm{E}-02$ \\
\hline \multirow[t]{5}{*}{ Feb. 28} & 2 & 10.64 & $7.40 \mathrm{E}+01 \pm 8.34 \mathrm{E}-02$ \\
\hline & 3 & 10.7 & $6.71 \mathrm{E}+01 \pm 7.92 \mathrm{E}-02$ \\
\hline & FI & 32.04 & $6.51 \mathrm{E}+01 \pm 4.71 \mathrm{E}-02$ \\
\hline & $\mathrm{FI}+\mathrm{BI}$ & 42.75 & $6.36 \mathrm{E}+01 \pm 4.00 \mathrm{E}-02$ \\
\hline & 0 & 10.3 & $5.87 \mathrm{E}+01 \pm 7.55 \mathrm{E}-02$ \\
\hline 4 & 1 & 10.28 & $5.30 \mathrm{E}+01 \pm 7.18 \mathrm{E}-02$ \\
\hline \multirow[t]{5}{*}{ Mar. 8} & 2 & 10.25 & $6.54 \mathrm{E}+02 \pm 7.99 \mathrm{E}-02$ \\
\hline & 3 & 10.3 & $6.44 \mathrm{E}+02 \pm 7.91 \mathrm{E}-01$ \\
\hline & FI & 30.85 & $6.24 \mathrm{E}+01 \pm 4.68 \mathrm{E}-02$ \\
\hline & $\mathrm{FI}+\mathrm{BI}$ & 41.13 & $5.99 \mathrm{E}+01 \pm 3.96 \mathrm{E}-02$ \\
\hline & 0 & 5.24 & $5.29 \mathrm{E}+01 \pm 1.01 \mathrm{E}-01$ \\
\hline 5 & 1 & 23.18 & $5.35 \mathrm{E}+01 \pm 4.80 \mathrm{E}-02$ \\
\hline \multirow[t]{5}{*}{ Mar. 15} & 2 & 5.24 & $6.02 \mathrm{E}+01 \pm 1.07 \mathrm{E}-01$ \\
\hline & 3 & 5.24 & $5.82 \mathrm{E}+01 \pm 1.05 \mathrm{E}-01$ \\
\hline & FI & 15.73 & $5.65 \mathrm{E}+01 \pm 6.39 \mathrm{E}-02$ \\
\hline & $\mathrm{FI}+\mathrm{BI}$ & 38.9 & $5.43 \mathrm{E}+01 \pm 3.91 \mathrm{E}-02$ \\
\hline & 0 & 15.37 & $4.45 \mathrm{E}+01 \pm 5.38 \mathrm{E}-02$ \\
\hline 6 & 1 & 21.65 & $4.64 \mathrm{E}+01 \pm 4.63 \mathrm{E}-02$ \\
\hline \multirow[t]{4}{*}{ Mar. 23} & 2 & 15.36 & $5.33 \mathrm{E}+01 \pm 5.89 \mathrm{E}-02$ \\
\hline & 3 & 15.36 & $5.04 \mathrm{E}+01 \pm 5.73 \mathrm{E}-02$ \\
\hline & FI & 52.37 & $4.91 \mathrm{E}+01 \pm 3.40 \mathrm{E}-02$ \\
\hline & $\mathrm{FI}+\mathrm{BI}$ & 67.74 & $4.81 \mathrm{E}+01 \pm 2.76 \mathrm{E}-02$ \\
\hline
\end{tabular}

from the Suzaku filtered event file, we used xselect - a HEAsoft tool. Before extracting spectra or light curves in the case of a point source, we had to create circular extraction region centered on the sources. It is recommended to create regions that encircle $99 \%$ of the point source flux. In later reduction processes we used the xissimarfgen script to build response files for our spectra. Xissimarfgen is a generator of auxiliary response files (ARFs) that is based on ray-tracing and that is designed for the analysis data from Suzaku XIS detectors. This script calculates ARFs through Monte-Carlo simulations. The idea is that the program ray-traces X-ray photons through the XRT and XIS and counts the number of events detected in extraction regions defined by user. We need to assume a sufficient number of photons to limit statistical errors to good level. In our analysis we assumed a number of simulated photons to 400000 to minimize Poisson noise in ARFs. To carry out further analysis, we downloaded redistribution matrix files (RMFs) from CALDB catalogs (for appropriate dates).

Since we have created ARFs and collected all RMFs files, we used an ASCA tool called addascaspec to combine source and 
background spectra and the responses of Suzaku XIS0, XIS2, and XIS3 (FI chip) data. We also tried to add all FI and BI (XIS1) chips even though such a procedure was not recommended, but we thought it can increase statistics in softer parts of the spectra.

We found out that over all six observations, the count rate declined with time. We know that there is a count rate threshold above which data from the source can suffer from a photon pileup effect. For a point source, it is $\sim 100$ counts/arcmin $2 / \mathrm{s}$ (CCD exposure) for the Suzaku XIS instrument. In all six observations, the count rate for $4 \mathrm{U} 1630-472$ was around 100 counts/s, so we can assume that the data have suffered from pile up or have not, since both are possible.

To minimize the pileup effect, we used two externally contributed routines, aeattcor.sl and pile_estimate.sl ${ }^{1}$. Both scripts are designed to analyze of Suzaku data that might be affected by the photon pile-up effect. Script aeattcor.sl improves the attitude correction for the slow wobbling of the optical axis for bright sources by using their detected image to create a new attitude file. Next, script pile_estimate.sl is designed to be run just after aeattcor.sl. It creates an image that will show an estimated, minimum pile-up fraction for user specified levels. This allows us to create a region file that excludes the most piled areas, and then estimate the effective pile-up fraction of the remaining events.

But data with a removed pile-up effect had much poorer statistics than those without removal, so in further modeling we did not used cleared data. 4U 1630-472 is near the threshold for pile-up, but we also can assume that there is no pile-up as done by Kubota et al. (2007). All other data reduction and calibration uncertainties were done in the same way as in that paper. Extracted XIS spectra for six observations and the region of iron absorption lines are presented in Fig. 1. The final exposure time and count rate in each XIS CCD chip for each epoch of observation is presented in Table 3.

\subsection{Spectral fitting}

Our numerical disk spectra (Różańska et al. 2011b) were converted to the FITS format, which is suitable for XSPEC fitting package (version 12.6). Atmospheric models, hereafter named ATM, were rare in parameter space. Since we do not know $M_{\mathrm{BH}}$, $\dot{m}$ and $a$ of the source, we took a prior set of parameters $\dot{m}$ or $a$ for a range of inclinations and fixed mass $M_{\mathrm{BH}}=10 M_{\odot}$. We did not aim here to derive the accretion rate or spin of the black hole. For the first time we fitted iron absorption line profiles different than simple Gaussian.

It was almost obvious that the best model is the hottest one, which was obtained for $\dot{m}=0.01$ in Edd. units and spin $a=0.98$, since EWs of the iron line were the biggest (see the two leftmost columns in Table 2). After that, we were able to fit observations by taking the viewing angle as the only free parameter.

\subsubsection{Continuum}

First, we performed fitting over the whole energy range 2-9 keV. Instead of using multitemperature XSPEC model DISKBB, we applied our atmospheric disk emission ATM multiplied by interstellar absorption WABS model. Additionally, we added the POWERLAW model to mimic the possible influence of irradiation at a hard part of the spectrum. From a theoretical point of view, in sources in soft state, which are dominated by disk emission, we expect that irradiation only slightly changes the hard tail of the disk spectrum. From an instrumental point of view,

\footnotetext{
1 http://space.mit.edu/ASC/software/suzaku/
}

the high-energy part can be affected by pile-up (see Sect. 4.2). In Kubota et al. (2007) this effect was modeled by a broad Gaussian in emission at $10 \mathrm{keV}$. In our fitting procedure, the POWERLAW model with flat photon index should balance those two effects. Following suggestions made by Kubota et al. (2007), we added a Gaussian at $\sim 3.2 \mathrm{keV}$ with width on the order of $0.1 \mathrm{keV}$ to reduce systematic errors arising from current uncertainties in the instrument response at the gold M-II edge.

We point out here that our ATM atmospheric disk emission deviates from the multiblackbody disk spectrum, since we took Compton scattering into account in hot disk atmosphere (Różańska et al. 2011a). We did not assume anything about the total disk hardening factor. The effect of spectrum hardening is self consistently computed in our numerical model.

There are two ways to obtain high effective temperature in the inner rings of the accretion disk. First, high $T_{\text {eff }}$ is the result of a high accretion rate. This is quite obvious because a higher accretion rate implies generation of higher radiation flux especially in the inner rings. Therefore, the value of accretion rate affects the most energetic part of the spectrum. The second way is to spin the black hole up. If the black hole rotates the inner rings radiate more energy, since the marginally stable orbit moves towards the black hole. Those rings are hot, and therefore, the total continuum spectrum looks as if the disk accreted at a high accretion rate. Additionally, disk spectra have slightly different shapes for various viewing angles. To distinguish which particular spectrum really fits the data, we need many more models. For 4U 1630-472, observed data favor the hottest model. However, we cannot claim anything about the accretion rate and black hole spin in this source. This is rather because absorption lines in such a model are the strongest, and they agree with observations.

Taking into account that the source $4 \mathrm{U} 1630-472$ is covered by interstellar gas of high column density on the order $8-9 \times$ $10^{22} \mathrm{~cm}^{-2}$, and the data were slightly affected by pile-up, we present continuum fitting only for Epoch 3 data. Every set of observations gave similar results.

Figure 2 presents our continuum fitting for Epoch 3 of FI+BI coadded spectra. The absorbing column density from interstellar gas was fitted to $N_{\mathrm{H}}=8.23 \pm 0.08$, which is consistent with previous fits. The best-fitted atmospheric model gives the inclination $i=11 \pm 5^{\circ}$ and normalization $1.50 \pm 0.08 \times 10^{-5}$. Additional POWERLAW photon index equals $\Gamma=1.48 \pm 0.55$. In this fitting we did not freeze any parameter. Our goodness of fit, i.e. $\chi^{2}$ per degrees of freedom, is $2618.57 / 2182=1.2$.

\subsubsection{Iron line complex}

For further analysis we decided to proceed by fitting the energy band restricted to the region of the iron line complex, i.e. between 6 and $9 \mathrm{keV}$. In that narrow band we still fit lines, together with the underlying continuum, as a single model, ATM. We did not make any additional assumption to the line or edge profile, since all features are already built up in our radiative transfer calculations of atmospheric disk emission.

Since back-illuminated XIS1 chip improves the statistics only in a low-energy tail around $2 \mathrm{keV}$ or less, it should cause no difference in the range between $6-9 \mathrm{keV}$. Therefore, for further analysis we only took spectra from front-illuminated XIS023 chips, as in the paper by Kubota et al. (2007).

Choosing the energy band between $6-9 \mathrm{keV}$ we reduce the number of degrees of freedom by a factor 2.5. We again fit WABS * (ATM + POWERLAW) and results of this fitting are presented in Fig. 3. The importance of the POWERLAW model is now lower than where fitting was done for the full energy band 


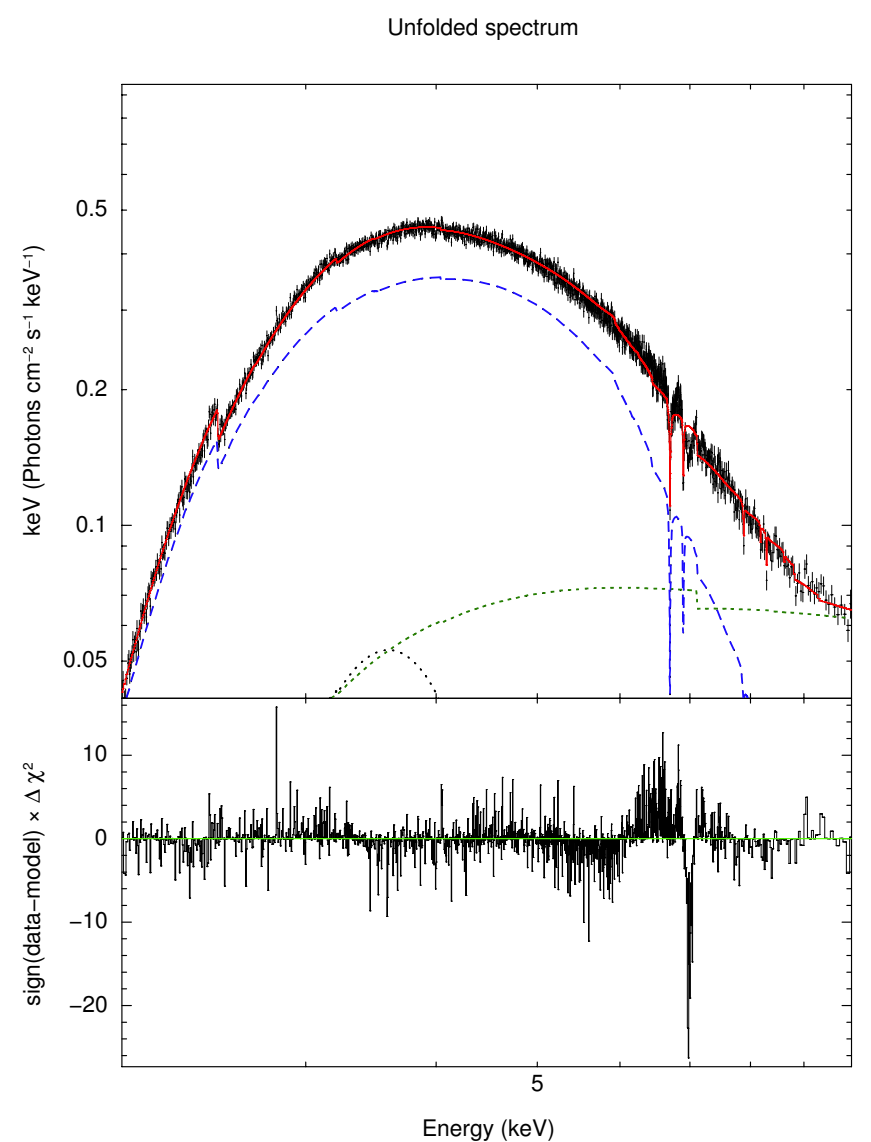

Fig. 2. Fitting of atmospheric disk model to the Epoch 3 data in the range $2-9 \mathrm{keV}$. The observed data, black crosses, are the sum of three front-illuminated (XIS0,2,3) and one back-illuminated (XIS1) CCDs. The total model WABS * (ATM+PL + GAUSSIAN) is represented by a red line. Separate model components are represented by: a long-dashed blue line - ATM, short-dashed green line - PL, and dotted black line instrumental GAUSSIAN. Reduced $\chi^{2} /$ d.o.f. $=2618.57 / 2182=1.2$ for the total model.

(see Sect. 4.3.1). The photon index is usually $-0.7 \pm 0.18$ and does not change much during different periods of observations. Similarly, the column density of galactic interstellar absorption $N_{\mathrm{H}}$ in most cases equals $8.16 \pm 0.12 \times 10^{22} \mathrm{~cm}^{-2}$. Since interstellar absorption should not change in different epochs, we fixed this parameter at $8 \times 10^{22} \mathrm{~cm}^{-2}$. Only for observation number 6 we did allow interstellar absorption to vary, but the fit is still with good statistics. We show all fitting parameters in Tables 4 and 5 .

Again, all six spectra imply the inclination angle $i=11 \pm 5^{\circ}$, since absorption lines in the disk seen "face on" are the deepest in our model. Nevertheless, we have checked that the disk observed at the viewing angle $i=70^{\circ}$, appropriate for dipping sources, can also explain observations. Surprisingly, fitting results with inclination frozen at $70^{\circ}$ also give quite good statistics (see Table 5).

The most important results of this paper are presented in Fig. 4, which is the enlarged part of Fig. 3. Resonant helium and hydrogen-like iron lines are clearly seen with their complex profiles. Our absorption lines do not need any velocity shift, unlike in the previous analysis (Kubota et al. 2007). Absorption lines from the upper parts of static accretion disk atmosphere can also explain observations satisfactorily.
Table 4. Fitting results of our atmospheric disk emission model to the 4U 1630-472 Suzaku data between 6-9 keV for the inclination angle $i=11 \pm 5^{\circ}$.

\begin{tabular}{lllll}
\hline \hline Ep. & WABS & ATM & PL & $\chi^{2} /$ d.o.f. \\
& $N_{\mathrm{H}}\left(10^{22}\right)$ & $\begin{array}{l}\text { Inc. }(d e g) \\
\text { Norm }\left(10^{-5}\right)\end{array}$ & $\begin{array}{l}\text { Ph. index } \\
\text { Norm }\left(10^{-3}\right)\end{array}$ \\
\hline 1 & 8 & $11 \pm 5$ & -0.7 & $2198.07 / 819$ \\
& & $2.9 \pm 0.6$ & $3.46 \pm 0.07$ & $=2.68$ \\
\hline 2 & 8 & $11 \pm 5$ & -0.7 & $1093.33 / 819$ \\
& & $2.04 \pm 0.02$ & $1.70 \pm 0.04$ & $=1.33$ \\
\hline 3 & 8 & $11 \pm 5$ & -0.7 & $1212.85 / 819$ \\
& & $1.96 \pm 0.48$ & $1.67 \pm 0.06$ & $=1.48$ \\
\hline 4 & 8 & $11 \pm 5$ & -0.7 & $1241.95 / 819$ \\
& & $1.76 \pm 0.46$ & $1.38 \pm 0.06$ & $=1.52$ \\
\hline 5 & 8 & $11 \pm 5$ & -0.7 & $793.06 / 819$ \\
& & $1.17 \pm 0.48$ & $0.94 \pm 0.07$ & $=0.97$ \\
\hline 6 & $7.82 \pm 0.98$ & $11 \pm 5$ & $-0.24 \pm 0.27$ & $995.85 / 817$ \\
& & $1.20 \pm 0.47$ & $3.16 \pm 0.2$ & $=1.22$ \\
\hline
\end{tabular}

Table 5. Fitting results of our atmospheric disk emission model to the 4U 1630-472 Suzaku data between 6-9 keV for the inclination angle $i=70 \pm 6^{\circ}$.

\begin{tabular}{lllll}
\hline \hline Ep. & WABS & ATM & PL & $\chi^{2} /$ d.o.f. \\
& $N_{\mathrm{H}}\left(10^{22}\right)$ & $\begin{array}{l}\text { Inc. }(\text { deg }) \\
\text { Norm }\left(10^{-5}\right)\end{array}$ & $\begin{array}{l}\text { Ph. index } \\
\text { Norm }\left(10^{-3}\right)\end{array}$ \\
\hline 1 & 8 & $70 \pm 6$ & -0.7 & $2236.77 / 819$ \\
& & $4.78 \pm 0.03$ & $3.59 \pm 0.03$ & $=2.73$ \\
\hline 2 & 8 & $70 \pm 6$ & -0.7 & $1101.27 / 819$ \\
& & $3.347 \pm 0.032$ & $1.79 \pm 0.04$ & $=1.34$ \\
\hline 3 & 8 & $70 \pm 6$ & -0.7 & $1262.26 / 819$ \\
& & $3.253 \pm 0.024$ & $1.76 \pm 0.03$ & $=1.54$ \\
\hline 4 & 8 & $70 \pm 6$ & -0.7 & $1290.14 / 819$ \\
& & $2.915 \pm 0.023$ & $1.46 \pm 0.03$ & $=1.57$ \\
\hline 5 & 8 & $70 \pm 6$ & -0.7 & $796.61 / 819$ \\
& & $1.957 \pm 0.024$ & $1.00 \pm 0.03$ & $=0.97$ \\
\hline 6 & $7.37 \pm 0.96$ & $70 \pm 6$ & $-0.7 \pm 0.18$ & $1033.43 / 817$ \\
& & $2.035 \pm 0.093$ & $1.20 \pm 0.08$ & $=1.26$ \\
\hline
\end{tabular}

\section{Conclusions}

In this paper we presented fitting of complex continuum and line numerical models to X-ray spectra of 4U 1630-472. In our models the spectrum of disk emission was obtained from careful radiative transfer computations including Compton scattering on free electrons. The Fe line profiles are computed as the convolution of natural, thermal, and pressure broadening mechanisms. The advantage of our models is that the continuum is fitted with lines simultaneously, which has never been done before in the analysis of X-ray absorption lines seen in LMXBs. The usual procedure is to fit both the disk emission as a standard model in XSPEC fitting package, and Gaussian lines, where the energy of line centroid is a free parameter of fitting. In such a case, lines are usually blueshifted indicating that absorbing matter outflows. We show in this paper, that in the case of 4U 1630-472 there is enough to assume zero velocity shift of absorbing matter. In our paper, iron absorption lines originate in the hot atmosphere above the accretion disk. 

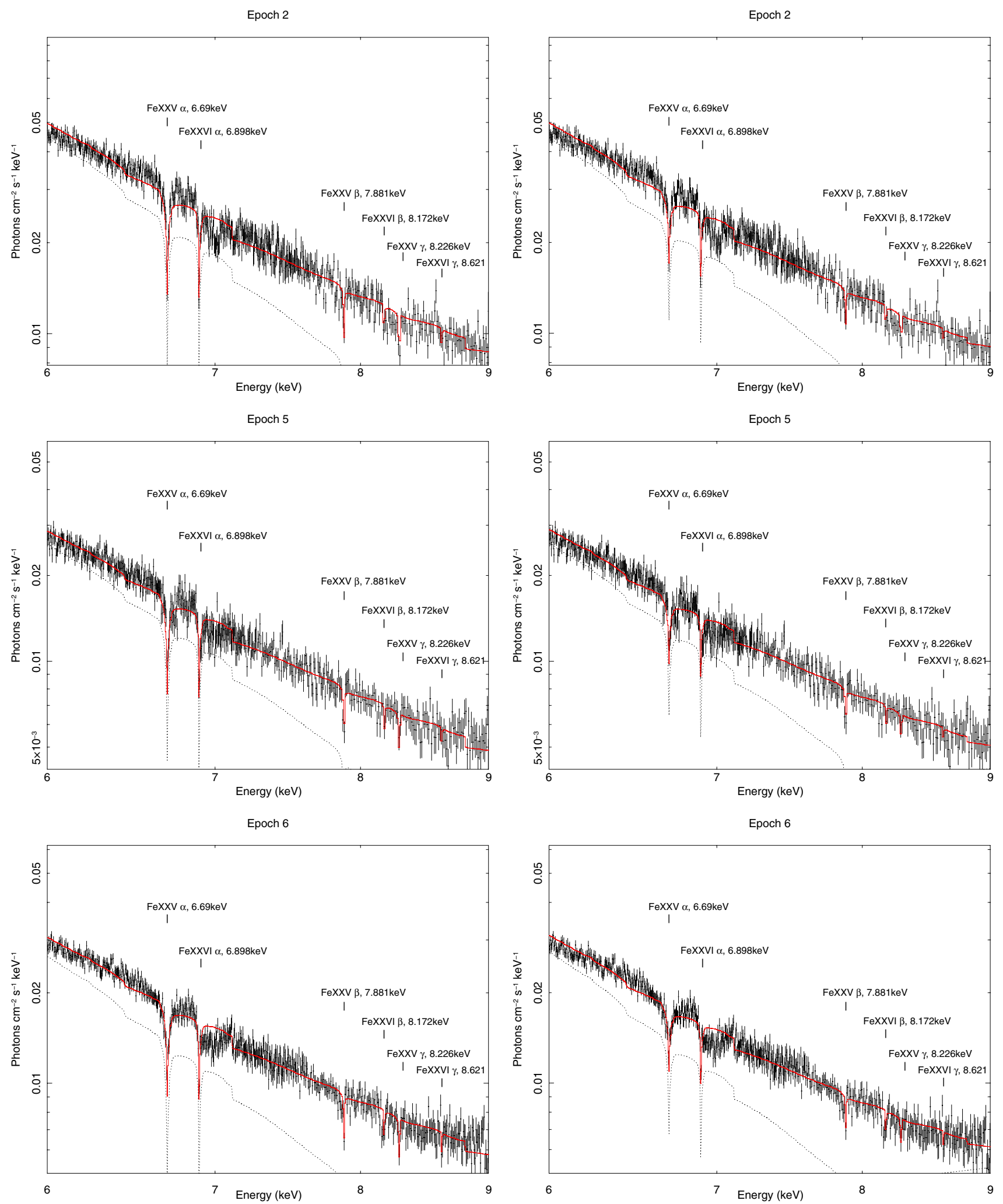

Fig. 3. Iron line region from 6-9 keV, where absorption features are clearly seen. The various panels represent observations for three different epochs. Black crosses show the data, dotted black lines represent ATM model, while solid red lines show the total model. Upper panels show the disk seen at $i=11 \pm 5^{\circ}$, whereas lower panels show the disk seen at $i=70 \pm 6^{\circ}$. All spectra favor fitting for $i=11 \pm 5^{\circ}$, since modeled lines are the deepest for this model.

Our models are only parametrized by the mass and spin of the black hole, and the disk accretion rate constant at all radii. Since our computations are very time consuming and we have only few collected models, we are not able to derive those parameters from continuum fitting. On the other hand, the quality of the Suzaku data is high enough to follow the shape of continuum even in heavily obscured sources (for 4U 1630-472, obscuration due to Galactic absorption equals ca. $8 \times 10^{22} \mathrm{~cm}^{-2}$ ). 
A. Różańska et al.: Disk emission and atmospheric absorption lines in black hole candidate 4U 1630-472
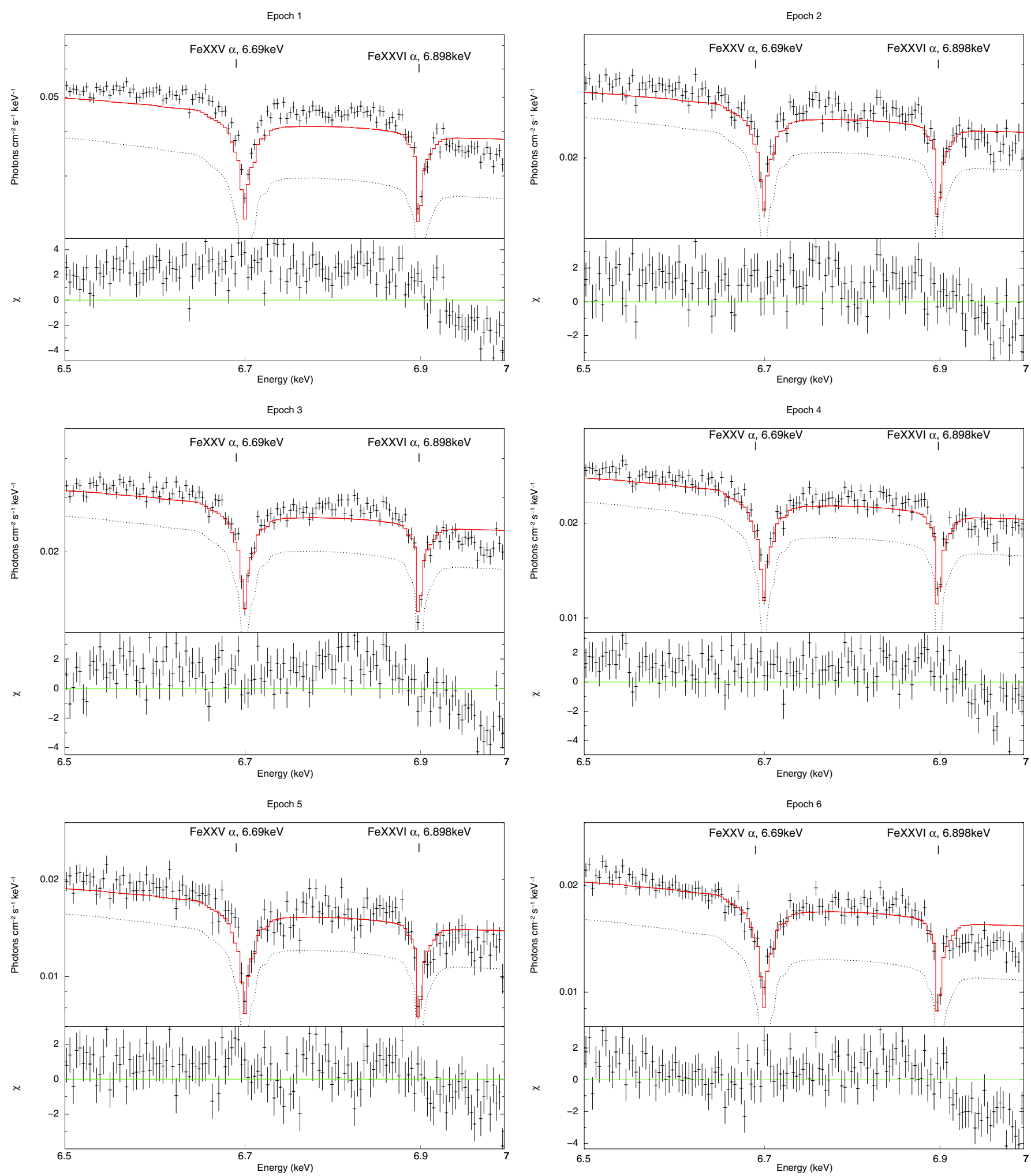

Fig. 4. Focus on the resonant $\mathrm{He}$ and $\mathrm{H}$-like iron lines for the fitting done in the 6-9 keV energy range (see text for explanation). Black crosses show the data, dotted black lines represent the ATM model, while solid red lines show the total model. Clearly, complicated line profiles computed in our model match the observations very well for almost every epoch. Residues are presented below each panel. Here we present spectra for $i=11 \pm 5^{\circ}$.

The accretion-disk atmosphere spectra fit the Suzaku data for 4U 1630-472 very well. The best fit we have obtained for the inclination angle $i=11^{\circ}$. For higher angles, i.e. $i=70^{\circ}$, the fit is just slightly worse. This angle is within the range of inclination suggested in the literature when taking dipping behavior into account and assuming absorption in the wind. The small difference in fit quality between different inclinations does not allow us to claim constraints on $i$.

Of course, models such as DISKBB+PL plus absorption with two Gaussians always show better statistics than our more complex model, But the former model has many free parameters. It is a general rule that the more free parameters we have, 
the more accurate the final fit. Our model has no free parameters connected with lines, therefore, it has at least six parameters less than the set DISKBB+PL plus two Gaussians applied by Kubota et al. (2007). According to any global disk models considered for the mass of central object on the order of $10 M_{\odot}$ or less, the effective temperature of the inner radii reaches $10^{7} \mathrm{~K}$. In such a case, the possible origin of absorption lines from accretion disk atmospheres should be taken into account when modeling the winds in X-ray binaries.

In the wind theory, it is widely accepted that the wind can be launched at the accretion disk surface. Upper layers of atmosphere can become unstable and start to blow material out owing to the radiation pressure. Our analysis does not contradict this fact, but instead shows that absorption at upper atmospheric layers cannot be distinguished from the absorption in the wind, which may be launched in the same region.

In this work we do not aim to tightly constrain parameters of the object but rather to show that emission from the accretion disk atmosphere is an important mechanism that gives a vital explanation or at least part of an answer to the question of the origin of iron absorption in X-ray binaries.

The major conclusion of our analysis is that the data of $4 \mathrm{U} 1630-472$ are interpreted well by the disk-shaped emission and that absorption lines do not need to set any velocity shift to explain data. Therefore, the wind explanation for absorbing matter is questionable and not unique. We showed that X-ray data of current quality can be interpreted in several ways and we cannot easily solve this ambiguity. We conclude that the wind theory can be an artefact of the fitting procedure, when the continuum and lines are fitted as separate model components. Data with higher spectral resolution are needed to distinguish between the two models. Future satellites with calorimeters, such as ASTRO$\mathrm{H}$ or Athena+, will yield the answer.

Acknowledgements. This research was supported by Polish Ministry of Science and Higher Education grant No. N N203 511638, and by Polish National Science Center grant No. 2011/03/B/ST9/03281. It received funding from the European Union Seventh Framework Program (FP7/2007-2013) under grant agreement No.312789.

\section{References}

Abramowicz, M. A., Czerny, B., Lasota, J. P., \& Szuszkiewicz, E. 1988, ApJ, 332, 646

Boirin, L., \& Parmar, A. N. 2003, A\&A, 407, 1079

Boirin, L., Parmar, A. N., Barret, D., Paltani, S., \& Grindlay, J. E. 2004, A\&A, 418, 1061

Brandt, W. N., \& Schulz, N. S. 2000, ApJ, 544, L123

Church, M. J., Reed, D., Dotani, T., Bałucińska-Church, M., \& Smale, A. P. 2005, MNRAS, 359, 1336

Díaz Trigo, M., Sidoli, L., Boirin, L., \& Parmar, A. N. 2012, A\&A, 543, A50

Frank, J., King, A. R., \& Lasota, J.-P. 1987, A\&A, 178, 137

Griem, H. R. 1974, Spectral line broadening by plasmas (New York: Academic Press)

Jones, C., Forman, W., Tananbaum, H., \& Turner, M. J. L. 1976, ApJ, 210, L9

Kallman, T., \& Bautista, M. 2001, ApJS, 133, 221

King, A. L., Miller, J. M., Raymond, J., et al. 2012, ApJ, 746, L20

Kotani, T., Ebisawa, K., Dotani, T., et al. 2000, ApJ, 539, 413

Kubota, A., Dotani, T., Cottam, J., et al. 2007, PASJ, 59, 185

Kuulkers, E., Wijnands, R., Belloni, T., et al. 1998, ApJ, 494, 753

Lee, J. C., Reynolds, C. S., Remillard, R., et al. 2002, ApJ, 567, 1102

Madej, J., \& Różańska, A. 2004, MNRAS, 347, 1266

Miller, J. M., Raymond, J., Homan, J., et al. 2006, ApJ, 646, 394

Miller, J. M., Raymond, J., Reynolds, C. S., et al. 2008, ApJ, 680, 1359

Novikov, I. D., \& Thorne, K. S. 1973, in Black Holes (Les Astres Occlus), eds. C. Dewitt, \& B. S. Dewitt, 343

Orosz, J. A., \& Bailyn, C. D. 1997, ApJ, 477, 876

Parmar, A. N., Stella, L., \& White, N. E. 1986, ApJ, 304, 664

Parmar, A. N., Angelini, L., \& White, N. E. 1995, ApJ, 452, L129

Parmar, A. N., Oosterbroek, T., Boirin, L., \& Lumb, D. 2002, A\&A, 386, 910

Ponti, G., Fender, R. P., Begelman, M. C., et al. 2012, MNRAS, 422, L11

Różańska, A., \& Madej, J. 2008, MNRAS, 386, 1872

Różańska, A., Madej, J., \& Gancarczyk, M. 2011a, in Proc. Fast X-ray Timing and Spectroscopy at Extreme Count Rates (HTRS 2011), 20 available at http://pos.sissa.it/cgi-bin/reader/conf. cgi? confid=122

Różańska, A., Madej, J., Konorski, P., \& Sạdowski, A. 2011b, A\&A, 527, A47

Schnerr, R. S., Reerink, T., van der Klis, M., et al. 2003, A\&A, 406, 221

Shakura, N. I., \& Sunyaev, R. A. 1973, A\&A, 24, 337

Sidoli, L., Oosterbroek, T., Parmar, A. N., Lumb, D., \& Erd, C. 2001, A\&A, 379, 540

Tomsick, J. A., Lapshov, I., \& Kaaret, P. 1998, ApJ, 494, 747

Ueda, Y., Inoue, H., Tanaka, Y., et al. 1998, ApJ, 492, 782

Ueda, Y., Asai, K., Yamaoka, K., Dotani, T., \& Inoue, H. 2001, ApJ, 556, L87

White, N. E., \& Swank, J. H. 1982, ApJ, 253, L61 